\title{
Examination of experimental evidence of chaos in the bound states of ${ }^{208} \mathrm{~Pb}$
}

\author{
L. Muñoz, ${ }^{1, *}$ R. A. Molina, ${ }^{2}$ J. M. G. Gómez, ${ }^{1}$ and A. Heusler ${ }^{3}$ \\ ${ }^{1}$ Grupo de Física Nuclear, Facultad de Ciencias Físicas, Universidad Complutense, E-28040 Madrid, Spain \\ ${ }^{2}$ Instituto de Estructura de la Materia, IEM-CSIC, Serrano 123, Madrid, E-28006, Spain \\ ${ }^{3}$ Gustav-Kirchhoff-Str. 7/1, D-69120 Heidelberg, Germany
}

(Received 12 April 2016; revised manuscript received 30 June 2016; published 18 January 2017)

\begin{abstract}
We study the spectral fluctuations of the ${ }^{208} \mathrm{~Pb}$ nucleus using the complete experimental spectrum of 151 states up to excitation energies of $6.20 \mathrm{MeV}$ recently identified at the Maier-Leibnitz Laboratorium at Garching, Germany. For natural parity states the results are very close to the predictions of random matrix theory (RMT) for the nearest-neighbor spacing distribution. A quantitative estimate of the agreement is given by the Brody parameter $\omega$, which takes the value $\omega=0$ for regular systems and $\omega \simeq 1$ for chaotic systems. We obtain $\omega=0.85$ which is, to our knowledge, the closest value to chaos ever observed in experimental bound states of nuclei. By contrast, the results for unnatural parity states are far from RMT behavior. We interpret these results as a consequence of the strength of the residual interaction in ${ }^{208} \mathrm{~Pb}$, which, according to experimental data, is much stronger for natural than for unnatural parity states. In addition, our results show that chaotic and nonchaotic nuclear states coexist in the same energy region of the spectrum.
\end{abstract}

DOI: 10.1103/PhysRevC.95.014317

\section{INTRODUCTION}

The atomic nucleus is generally considered a paradigmatic case of quantum chaos. Intuitively one can expect that fastmoving nucleons interacting with the strong nuclear force and bound in the small nuclear volume should give rise to a chaotic motion. During the last three decades the quest for chaos in nuclei was quite intensive, both with theoretical calculations using nuclear models and with detailed analyses of experimental data. Statistical spectroscopy studies in nuclei have been also motivated by a desire to understand the implications of chaotic behavior in many-body quantum systems. Theoretical calculations, especially shell-model calculations, have shown a strongly chaotic behavior of bound states at higher excitation energy, in regions of high level density. However, as we discuss below, it was not possible up to now to observe chaos in the experimental bound energy levels of any single nucleus. For a comprehensive review of chaos in nuclei see, for example, Gómez et al. [1] and Weidenmüller and Mitchell [2].

In this paper we analyze spectral fluctuations in ${ }^{208} \mathrm{~Pb}$ using the experimental data recently obtained by Heusler et al. [3] from the study of ${ }^{208} \mathrm{~Pb}\left(\mathrm{p}, \mathrm{p}^{\prime}\right),{ }^{207} \mathrm{~Pb}(\mathrm{~d}, \mathrm{p})$, and ${ }^{208} \mathrm{~Pb}\left(\mathrm{~d}, \mathrm{~d}^{\prime}\right)$ reactions. The lowest 151 states have been identified with spin and parity assignments. This represents the largest ensemble known up to now which can be used for a statistical investigation of the chaotic behavior among bound states in an atomic nucleus. We have found sequences of bound states with clearly chaotic statistics, while other sequences exhibit intermediate properties between chaos and regularity. These two different behaviors are related to the strength of the residual interaction that destroys the mean-field order, as predicted by shell-model calculations. To our knowledge it is the first time that such a behavior is inferred directly from experimental nuclear states.

\footnotetext{
*1munoz@ucm.es
}

\section{CHAOS IN NUCLEI}

\section{A. Spectral fluctuations in experimental nuclear bound states}

For a quantum system like the atomic nucleus, which has no classical limit, the term chaos started to be used when Haq, Pandey, and Bohigas [4] analyzed the spectral fluctuations of a very large number of experimentally identified neutron and proton $J^{\pi}=1 / 2^{+}$resonances just above the one-nucleon emission threshold and showed that they agree very well with the spectral fluctuations of the Gaussian orthogonal ensemble (GOE) of random matrix theory (RMT). According to the BGS conjecture [5] the agreement with GOE is characteristic of quantum chaos. On the contrary, spectral fluctuations that coincide with those of a Poisson distribution are characteristic of a regular quantum system [6].

Thus, in this sense, it is clear that nuclei are very chaotic in the energy region just above the one-nucleon emission threshold. But for bound states, the situation is not so clear, because a good analysis of fluctuations in experimental energy spectra requires the knowledge of sufficiently long, pure, and complete sequences, i.e., with the same $J^{\pi} T$ values and without missing levels or $J^{\pi} T$ misassignments. But this ideal situation is rarely found in nuclei. For very light nuclei the number of bound levels is not sufficient for statistical purposes. For medium and heavy nuclei the identified levels are limited to the ground-state region, because at higher energy the level density becomes very high and the experimental identification of the energy and $J^{\pi}$ values becomes generally impossible.

Only in very few nuclei, namely ${ }^{26} \mathrm{Al}$ and ${ }^{30} \mathrm{P}$, the full experimental spectrum was essentially identified up to the proton separation energy at $E_{x} \simeq 8 \mathrm{MeV}$. A statistical analysis of level fluctuations in these nuclei was performed combining level spacings of different $J^{\pi}$ or $J^{\pi} T$ sequences [7]. A surprising result is that the behavior of the nearest neighbor spacing (NNS) distribution $P(s)$ and the behavior of the spectral rigidity statistic $\Delta_{3}(L)$ are quite similar in these nuclei whether or not the isospin $T$ is taken into account in the 
statistical analysis. As is well known, if a level sequence is not pure, i.e., contains states belonging to different symmetry classes, the strong level correlations characteristic of chaotic spectra are destroyed. Therefore one has to analyze separately the $J^{\pi}$ level sequences of different $T$. But both ${ }^{26} \mathrm{Al}$ and ${ }^{30} \mathrm{P}$ are odd-odd $N=Z$ nuclei, where states with isospin $T=0$ and $T=1$ have nearly equal density of levels in the ground-state region, and an isospin symmetry breaking of about $3 \%$ due to the Coulomb interaction may lead to similar fluctuation properties for $J^{\pi}$ or $J^{\pi} T$ sequences [8,9]. The isospin symmetry breaking seems to be responsible for the fact that the $P(s)$ statistic is equally far from GOE and Poisson predictions in these two nuclei.

Experimental pure level sequences in the ground-state region are generally too short for statistical analysis. But to improve statistics, level spacings from different nuclei can be combined into a single set to analyze the behavior of the NNS distribution $P(s)$. An extensive analysis of low-lying energy levels was performed by Shriner et al. [10] using experimental data along the whole nuclear chart. A total of 988 spacings from 60 different nuclei were included in the analysis. A simple quantitative measure of chaos or regularity is provided by the Brody parameter $\omega$, which in the extreme cases takes the value $\omega \simeq 1$ for $\mathrm{GOE}$ and $\omega=0$ for Poisson. For the whole set of 988 spacings the fit gives $\omega=0.43 \pm 0.05$, which is an intermediate value closer to Poisson than to GOE. Separating the data in six different mass regions a clear trend from GOE to Poisson is observed as the nuclear mass increases. For light nuclei with $A \leqslant 50$, the fit gives $\omega=0.72 \pm 0.16$, and for the heaviest nuclei with $A>230$ it gives $\omega=0.24 \pm 0.11$. Generally spherical nuclei are closer to GOE and deformed nuclei are closer to Poisson. The latter is not necessarily a manifestation of regular behavior for the low-lying states of these nuclei, because a deviation towards Poisson may be also due to the omission of some symmetry. In the present case of deformed nuclei it may be because of the omission of the $K$ quantum number, but it is not possible to quantify this effect with the available experimental data.

We may conclude that, for one reason or another, the analysis of fluctuations in experimental nuclear bound states has not shown the existence of clear chaotic motion, which should have a $P(s)$ distribution with $\omega$ close to 1 . By contrast, several analyses of level fluctuations have shown almost regular nuclear dynamics in deformed nuclei, where collective motion is dominant. In spherical nuclei experimental energy levels exhibit an intermediate behavior between GOE and Poisson, although more chaotic than regular, especially in light nuclei.

\section{B. Short-range level fluctuations in ${ }^{208} \mathrm{~Pb}$}

Experimental nuclear spectra become increasingly plagued with unidentified states, missing levels, and some misassignments as excitation energy increases. But we consider that the recent accurate data on ${ }^{208} \mathrm{~Pb}$ enable a meaningful statistical analysis of level fluctuations in a nucleus, with pure, complete, and reasonably long sequences.

Fluctuations are the departure of the actual level density from a local uniform density. Therefore it is essential to eliminate the smooth part of the exponential increase of the nuclear density, mapping the actual spectrum onto a quasiuniform spectrum with mean spacing $\langle s\rangle=1$. This step, called unfolding, is delicate and of utmost importance, because some of the unfolding procedures used in the literature can lead to completely wrong results on the behavior of level fluctuations [11]. In this work we have used the constant temperature formula [10],

$$
\bar{\rho}(E)=\frac{1}{T} \exp \left[\left(E-E_{0}\right) / T\right]
$$

where $T$ and $E_{0}$ are taken as parameters, and separate unfolding was performed for all $J^{\pi}$ sequences with a minimum of five known consecutive states. The longest sequence corresponds to the $J^{\pi}=3^{-}$states, with 19 consecutive levels. Gathering the unfolded spacings for all $J^{\pi}$ into a single set, there are 115 spacings.

An assessment of chaotic or regular behavior is given by comparison of the NNS distribution to Poisson and Wigner. The Poisson distribution is given by $P_{P}(s)=\exp (-s)$. The Wigner surmise, $P_{W}(s)=(\pi s / 2) \exp \left(-\pi s^{2} / 4\right)$, is a very good approximation to the GOE distribution.

A simple way to interpolate between the Poisson limit $(\omega=$ $0)$ and the Wigner surmise $(\omega=1)$ is provided by the Brody distribution,

$$
\begin{aligned}
P_{B}(s, \omega) & =(\omega+1) a_{\omega} s^{\omega} \exp \left(-a_{\omega} s^{\omega+1}\right), \\
a_{\omega} & =\left[\Gamma\left(\frac{\omega+2}{\omega+1}\right)\right]^{\omega+1},
\end{aligned}
$$

where $\Gamma$ is the gamma function. The Brody parameter $\omega$ is given by the best fit to the histogram of $P(s)$. When the number of spacings is not very large, it is preferable to fit the cumulative distribution,

$$
I(s)=\int_{0}^{s} P(x) d x .
$$

The cumulative Brody distribution is given by

$$
I_{B}(s, \omega)=1-\exp \left(-a_{\omega} s^{\omega+1}\right) .
$$

The fit to the full set of 115 experimental spacings gives $\omega=0.63 \pm 0.08$ for $P(s)$ and $\omega=0.68 \pm 0.02$ for $I(s)$. The two values are similar, but the cumulative fit is more accurate. Thus, from now on, we give the $\omega$ values for the cumulative distribution.

The relevant question with these results is how to interpret them regarding chaotic motion in ${ }^{208} \mathrm{~Pb}$. Because it is a double closed-shell nucleus, the excited states in the ground-state region have a somewhat simple structure, dominated by oneparticle one-hole (1p-1h) and a few $2 \mathrm{p}-2 \mathrm{~h}$ configurations. In fact Heusler et al. [3] have shown that the number of identified states at $E_{x}<6.20 \mathrm{MeV}$ nearly agrees with the number of states in this energy interval predicted by what they call the "extended schematic shell model," which is a simplified shell model consisting of $1 \mathrm{p}-1 \mathrm{~h}$ mean-field configurations plus the diagonal part of the surface delta interaction (SDI), extended with $2 \mathrm{p}-2 \mathrm{~h}$ configurations with SDI. Clearly the basis configurations are spread out among a small number of states; often only two or three configurations are dominant in each 
state. By contrast, GOE eigenstates are not dominated by any particular amplitudes. Thus the similarities with GOE are certainly limited. The result $\omega=0.68 \pm 0.02$ is similar to the value $\omega=0.72 \pm 0.16$ for nuclei with $A<50$ [10]. Therefore we may wonder whether it represents more or less a practical limit of possible chaos in nuclear bound states.

In the shell model the mean field gives rise to a regular motion and the residual interaction produces the mixing of basis states in the eigenstates, destroying the regular mean-field motion. This effect was observed in shell-model calculations introducing a strength parameter to modulate the residual interaction. As the strength parameter increases, the fluctuation measures of energy levels approach GOE behavior and thus the motion becomes chaotic [13].

\section{Comparison of short-range spectral fluctuations in natural and unnatural parity states}

Heusler et al. have shown [3] that there is better agreement of the extended schematic shell model with experiment for unnatural parity states $\left(J^{\pi}=0^{-}, 1^{+}, 2^{-}, \ldots, 11^{+}\right)$than for natural parity states $\left(J^{\pi}=0^{+}, 1^{-}, 2^{+}, \ldots, 12^{+}\right)$in ${ }^{208} \mathrm{~Pb}$. The excitation energies of 70 states with unnatural parity at $E_{x}<6.20 \mathrm{MeV}$ agree within about $0.2 \mathrm{MeV}$ with $1 \mathrm{p}-1 \mathrm{~h}$ configurations of the extended schematic shell model. By contrast, the excitation energies of about 20 natural parity states are more than $0.5 \mathrm{MeV}$ lower than the model prediction. Hence the residual interaction is much larger for natural than for unnatural parity states.

To check if this effect can be observed in the fluctuation measures, we have analyzed separately the NNS distribution of experimental natural and unnatural parity states. Table I shows the number of unfolded spacings of each type at $E_{x}<6.20$ $\mathrm{MeV}$ in ${ }^{208} \mathrm{~Pb}$ and the corresponding values of the Brody parameter obtained from the fit of the cumulative distribution, as well as the root-mean-square deviation (RMSD) of the experimental $I(s)$ from the Wigner and Poisson limits. Figure 1 shows the distributions $I(s)$ and $P(s)$ for natural and unnatural parity states, compared to Wigner and Poisson. To guide the eye we have also plotted the Brody distribution.

For natural parity states the behavior is definitely chaotic, with $\omega=0.85 \pm 0.02$. A bootstrap estimation of the error bar gives comparable results, $\omega=0.85_{-0.03}^{+0.05}$; see Appendix. The Brody and Wigner curves for $P(s)$ nearly coincide, and the

TABLE I. Number of spacings, Brody parameter $\omega$, and rms deviation from Wigner and Poisson distributions for different combinations of parity in the experimental states of ${ }^{208} \mathrm{~Pb}$ at $E_{x}<6.20 \mathrm{MeV}$.

\begin{tabular}{lccc}
\hline \hline \multirow{2}{*}{ Parity } & \multicolumn{3}{c}{ Number of spacings } \\
\cline { 2 - 4 } & All & Natural P. & Unnatural P. \\
\hline Even & 45 & 29 & 16 \\
Odd & 70 & 42 & 28 \\
Any & 115 & 71 & 44 \\
\hline Brody $\omega$ & $0.68 \pm 0.02$ & $0.85 \pm 0.02$ & $0.43 \pm 0.03$ \\
$(\text { RMSD })_{W}$ & 0.040 & 0.025 & 0.077 \\
$(\text { RMSD })_{P}$ & 0.115 & 0.129 & 0.088 \\
\hline \hline
\end{tabular}

dots representing the cumulative distribution clearly follow the Wigner distribution. To our knowledge, this is the closest GOE behavior ever observed in experimental nuclear bound states. It is worth commenting that the Wigner surmise is only a good analytical approximation to the GOE distribution, and that the best fit of $P_{B}(s, \omega)$ to the exact $P(s)$ distribution for GOE is obtained for $\omega=0.957$, not for $\omega=1$ [12].

By contrast, for unnatural parity states the cumulative distribution is intermediate between the two extremes, somewhat closer to Poisson, with $\omega=0.43 \pm 0.03$. There are only 44 spacings and the histogram of $P(s)$ oscillates a lot; see Appendix. But notice especially the different behavior of $P(s)$ for small spacings in natural and unnatural parity states. Level repulsion is seen to be much stronger for natural parity states. Strong level repulsion is characteristic of chaotic (Wigner-like) spectra, whereas for the regular motion $P(s)$ is maximal for small spacings.

We have also analyzed the NNS distributions for all the even and odd parity states. The Brody parameter is $\omega=0.61 \pm 0.05$ for positive parity and $\omega=0.67 \pm 0.04$ for negative parity. Hence, we do not observe any significant difference between even and odd parity.

We should keep in mind that the experimental $J^{\pi}$ sequences in nuclei are generally quite short and that any missing levels or misassignments always bias the statistical measures towards Poisson. The same kind of bias is quickly produced by some broken symmetry or approximate symmetry in the nuclear states. Therefore intermediate results in the statistical fluctuation measures should be taken with caution. But in the case of ${ }^{208} \mathrm{~Pb}$ all the states with $E_{x}<6.20 \mathrm{MeV}$ are now well identified, except for one or two tentative $J^{\pi}$ assignments at the upper end of the $5^{+}, 7^{+}$, and $8^{+}$states. We have checked that they essentially do not affect our results. Hence we are confident that the NNS distribution is not biased by missing levels or misassignments. The results reflect the degree of chaos caused by the residual interaction, and this is clearly seen comparing the NNS distributions of natural and unnatural parity states.

\section{Discussion}

Another important statistic for the assessment of chaos is the Dyson-Metha $\Delta_{3}(L)$, which measures long-range correlations. Unfortunately the available experimental $J^{\pi}$ sequences in ${ }^{208} \mathrm{~Pb}$ are too short for a reliable $\Delta_{3}(L)$ analysis. The longest known sequence is the $193^{-}$states, and the best we can say is that $\Delta_{3}(L)$ up to $L=6$ is nearly compatible with GOE behavior; see Appendix.

Let us briefly discuss now some theoretical results on chaos in nuclear bound states. Theoretical calculations provide long sequences of $J^{\pi}$ or $J^{\pi} T$ levels suitable for statistical analysis of fluctuations (no missing levels, no uncertain spin and parity assignments). Calculations performed with the spherical shell model, the cranking model, the interacting boson model, and other models have shown examples of highly regular energy spectra in deformed nuclei and examples of highly chaotic spectra in spherical nuclei, although there are exceptions in some nuclei. 

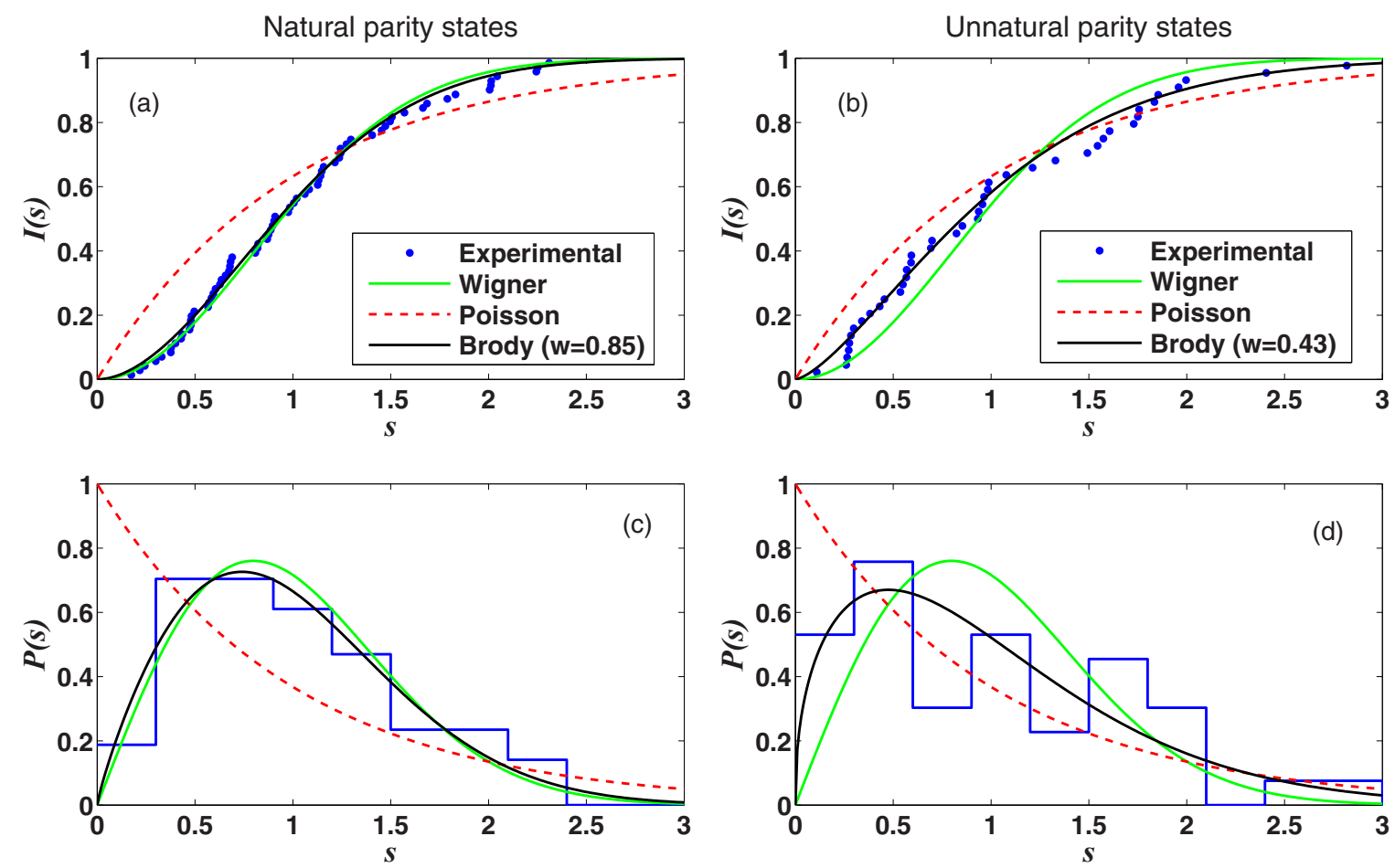

FIG. 1. Comparison of spectral fluctuation statistics for natural parity states (left panels) and unnatural parity states (right panels) in ${ }^{208} \mathrm{~Pb}$. (a) and (b) The cumulative nearest neighbor spacing (cumulative NNS) distribution for experimental levels (dots). (c) and (d) The NNS distribution $P(s)$ for experimental levels (histogram). In all panels the green (light gray) line is the Wigner surmise, the red dashed line is the Poisson distribution, and the black solid line is the best fit Brody distribution.

In spherical nuclei, where the shell model is most appropriate, many calculations have shown that for large configuration spaces, with $J^{\pi} T$ level sequences up to several thousand, the usual fluctuation measures $P(s)$ and $\Delta_{3}(L)$ agree very well with GOE predictions. To mention just some examples, we highlight the work of Zelevinsky et al. [13] in $2 s 1 d$ shell nuclei. In the middle of the $s d$ shell, ${ }^{28} \mathrm{Si}$ has 12 valence nucleons and the $J^{\pi} T$ shell-model Hamiltonian matrices have large dimensionalities and the agreement with GOE is excellent.

In the $2 p 1 f$ shell the configuration space and the level density are much larger than in $s d$-shell nuclei. Shell-model calculations with a realistic interaction have been performed to investigate the degree of chaos in different isotopes as a function of excitation energy by Molina et al. [14]. For example, in ${ }^{46} \mathrm{Sc}$ a total of 25498 spacings are included in the calculations, ensuring excellent statistics. The fluctuation statistics are in very good agreement with GOE, even for the low-lying levels above the yrast line, for all the Sc isotopes studied. In ${ }^{46} \mathrm{Ti}$, an even-even nucleus, with lower density of states in the ground-state region, the agreement with GOE is excellent as well, even at low excitation energies. However, for $\mathrm{Ca}$ isotopes the results are quite different. The Brody parameter is always smaller in $\mathrm{Ca}$ than in $\mathrm{Sc}$ in all isotopes and energy regions, and also when the full spectrum is considered. Furthermore, at low energies the fluctuations are more regular than chaotic, for instance, $\omega=0.25$ for the levels up to $5 \mathrm{MeV}$ above yrast in ${ }^{52} \mathrm{Ca}$. Similar results were obtained for $\mathrm{Pb}$ isotopes, with only valence neutrons outside the ${ }^{208} \mathrm{~Pb}$ core [15].
These examples of shell-model calculations with a realistic interaction illustrate the same phenomenon that makes the difference in the chaotic vs regular behavior of natural and unnatural parity states observed in the experimental energy levels of ${ }^{208} \mathrm{~Pb}$. The shell-model residual nn interaction is much weaker than the residual pn interaction. Ca isotopes have only neutrons in the $p f$ shell, but if just one neutron is replaced by a proton, the pn interactions destroy the mean-field order. Therefore Sc or Ti isotopes, having both protons and neutrons in the valence space of the $p f$ shell, exhibit strong chaotic characteristics even in the ground-state region.

\section{CONCLUSIONS}

In conclusion, the recent identification with spin and parity assignment of all the 151 states at $E_{x}<6.20 \mathrm{MeV}$ in ${ }^{208} \mathrm{~Pb}$ by Heusler et al. [3] has provided exceptionally long $J^{\pi}$ sequences of consecutive states free of missing levels and misassignments, enabling us to perform a reliable analysis of spectral fluctuations in this nucleus. Comparison of the experimental spectrum with extended $1 \mathrm{p}-1 \mathrm{~h}$ schematic shell-model calculations clearly indicate that the residual interaction is much stronger for natural than for unnatural parity states [3]. Therefore we have analyzed separately the spectral fluctuations of those two sets of states and have found that they behave very differently. The natural parity states exhibit results close to GOE and the unnatural parity states are far from GOE behavior. Thus these results clearly indicate that chaotic and nonchaotic states coexist in the energy region 
from the ground state up to $6.20 \mathrm{MeV}$ excitation energy [the neutron threshold in ${ }^{208} \mathrm{~Pb}$ is $\left.S(\mathrm{n})=7.368 \mathrm{MeV}\right]$. Furthermore, our analysis of the experimental spectrum has confirmed, to our knowledge for the first time, a well-known shell-model prediction, namely that chaos in nuclei arises when the residual interaction is strong enough to destroy the ordered motion of nucleons in the nuclear mean field.

\section{ACKNOWLEDGMENTS}

This research was conducted with support of Spanish Ministry of Science and Innovation Grants No. FIS2012-35316 and No. FIS2012-34479, MINECO/FEDER Grant No. FIS201563770-P, and CAM research consortium QUITEMAD+ S2013/ICE-2801.

\section{APPENDIX}

\section{Calculation of the $\Delta_{3}$ statistic}

Long-range statistical correlations in the spectra are very important measures for a complete characterization of the properties of chaotic and regular systems. Unfortunately, because of their own nature they need very long spectral sequences to provide meaningful results. The $\Delta_{3}$ is one of the most widely used long-range statistics and was defined originally by Dyson and Mehta in Ref. [16]. It is defined by

$$
\Delta_{3}(L)=\left\langle\min _{A, B} \frac{1}{L} \int_{E_{i}}^{E_{i}+L} d E^{\prime}\left[N\left(E^{\prime}\right)-A E^{\prime}-B\right]^{2}\right\rangle,
$$

where the angle brackets denote the spectral average over the values of $E_{i}$, the location of the window of $L$ levels within the spectrum. The parameters $A$ and $B$ are recalculated for each value of $i$. For a regular system with no level repulsion $\Delta_{3}(L)=L / 15$. The result for GOE spectra cannot be computed analytically but it is possible to obtain an asymptotic result valid for large $L$.

$$
\Delta_{3}(L)=\frac{1}{\pi^{2}}(\log L-0.0678) .
$$

An important result that can be obtained for GOE is the ensemble variance which is independent of $L, \sigma_{e}=0.110$ [16]. The Poisson estimate of the spectral variance of $\Delta_{3}(L)$ depends on $L, \sigma_{e}=0.11 \sqrt{L / N}[16,19]$. This value is high compared to the average value of the $\Delta_{3}(L)$ for small values of the sample size $N$. In fact, a rigorous study of the effect of small sample sizes on the statistical analysis of eigenvalue distributions concluded that $\Delta_{3}(L)$ was unreliable, and the most reliable statistic was the nearest-neighbor spacing distribution [17].

More recent analyses of the statistical usefulness of the $\Delta_{3}$ statistics for finding missing or spurious levels, for example, have been performed in $[18,19]$. They had more optimistic conclusions, but in their work the $\Delta_{3}$ statistic was never applied nor initially designed for being applied to level sequences of less than 40-50 consecutive levels.

For the practical calculation of $\left\langle\Delta_{3}(L)\right\rangle$ we have implemented the prescription described in Ref. [20]. It must

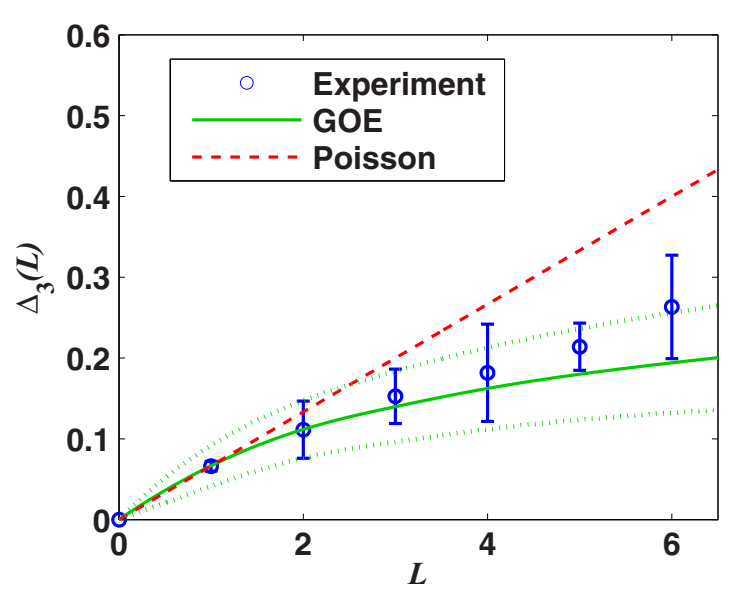

FIG. 2. Average $\Delta_{3}$ statistic as a function of the level distance $L$ calculated for the 19 experimental $J^{\pi}=3^{-}$states of ${ }^{208} \mathrm{~Pb}$ compared with GOE (solid line) and Poisson (dashed line) results. The dotted lines correspond to one standard deviation for the Poisson estimate of the spectral variance of $\Delta_{3}$ for GOE $[18,19]$.

be noted that to have a meaningful spectral average it is important to calculate $\Delta_{3}(L)$ through independent intervals of length $L$ in the spectrum. The number of such intervals limits the maximum value of $L$ for which the $\Delta_{3}(L)$ can be calculated [20]. In the case of ${ }^{208} \mathrm{~Pb}$ the largest sequence of levels with the same quantum numbers is the one for $J^{\pi}=3^{-}$ which contains 19 consecutive levels. We have calculated $\Delta_{3}(L)$ up to $L_{\max }=6$ and the results are shown in Fig. 2. The error bar shown for each point is just the error of the spectral average from the averaging over the different intervals in the spectrum. Taking into account the previous discussion, the information that can be obtained from these calculations is that the $\Delta_{3}(L)$ results for the $J^{\pi}=3^{-}$states are more or less compatible with GOE but not with Poisson spectra. For other $J^{\pi}$ states the sequences are too short for meaningful $\Delta_{3}(L)$ calculations.

\section{Quality of the fits and error estimation of the Brody parameter}

In Fig. 3 a series of panels shows the quality of the fits both for natural parity and for unnatural parity states. It is clear from these figures that the fits are of good quality and, as a consequence, the error of the $\omega$ parameter is relatively small.

Error estimation is difficult in the case of nonlinear fits. However, in the case of fits of a single parameter, like the ones we needed to estimate the Brody parameter in this work, the problem is easier and there are no major technical difficulties. The error quoted in the main text is the result of a standard $\chi^{2}$ calculation. We have also used a bootstrap procedure to calculate the error. The error is higher but it does not change any conclusion in the main text. The results of the Brody parameter using the error calculated with a bootstrap procedure are $\omega=0.43_{-0.04}^{+0.05}$ for the unnatural parity states and $\omega=0.85_{-0.03}^{+0.05}$ for the natural parity states. 

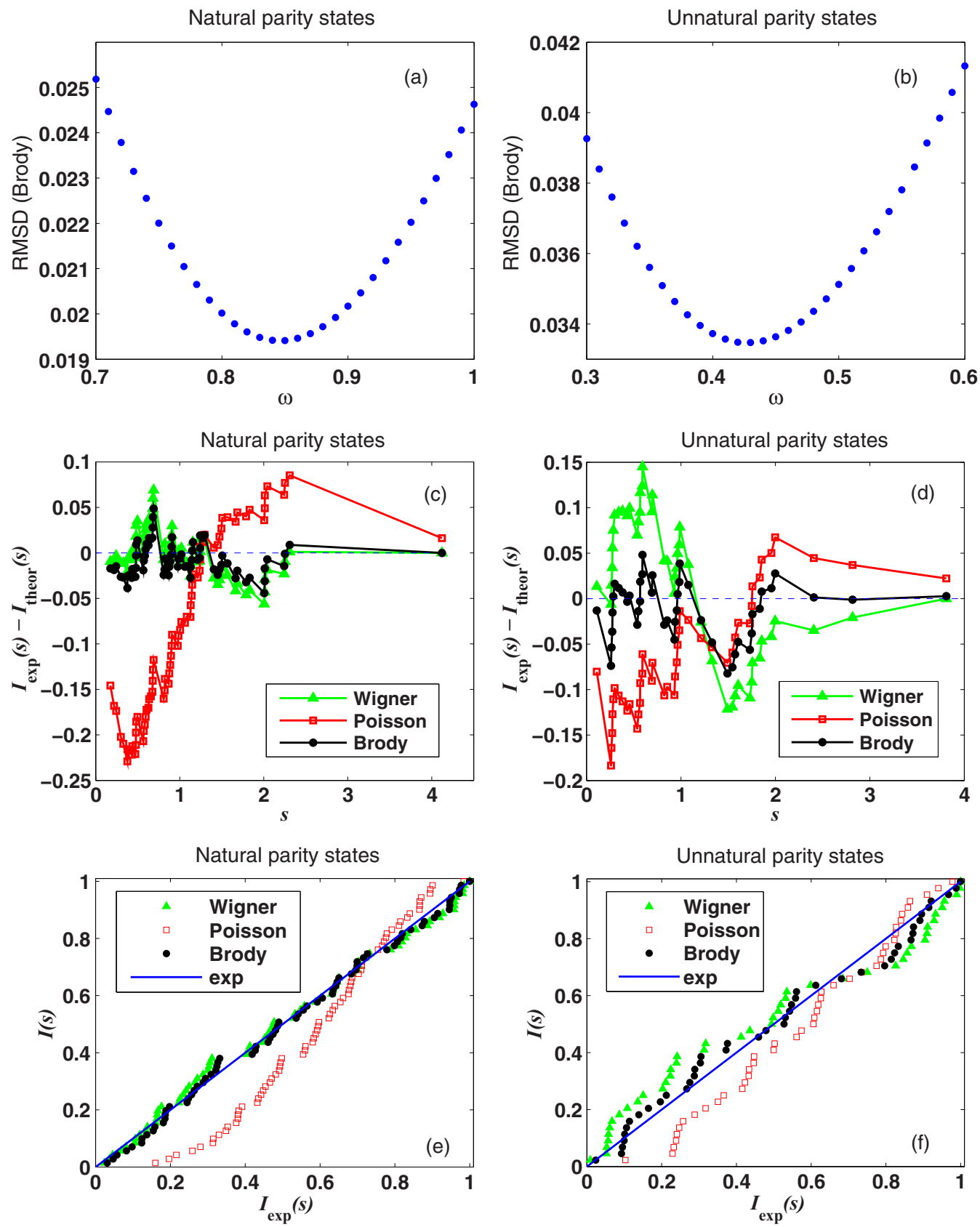

FIG. 3. Study of the quality of the fits presented in the paper, and comparison of results for natural parity states (left panels) and unnatural parity states (right panels) in ${ }^{208} \mathrm{~Pb}$. (a) and (b) The root-mean-square deviation (RMSD) of the experimental cumulative NNS distribution $I_{\text {exp }}(s)$ from the cumulative Brody distribution $I_{B}(s, \omega)$, as a function of the Brody parameter $\omega$. (c) and (d) The differences of $I_{\text {exp }}(s)$ with the cumulative distributions for Wigner (triangles), Poisson (squares), and the best Brody fit (filled circles). Similarly, panels (e) and (f) show all the cumulative distributions $I(s)$ in a linearized plot.

[1] J. M. G. Gómez, K. Kar, V. K. B. Kota, R. A. Molina, A. Relaño, and J. Retamosa, Phys. Rep. 499, 103 (2011).

[2] H. A. Weidenmüller and G. E. Mitchell, Rev. Mod. Phys. 81, 539 (2009).

[3] A. Heusler, R. V. Jolos, T. Faestermann, R. Hertenberger, H.-F. Wirth, and P. von Brentano, Phys. Rev. C 93, 054321 (2016).

[4] R. U. Haq, A. Pandey, and O. Bohigas, Phys. Rev. Lett. 48, 1086 (1982).
[5] O. Bohigas, M. J. Giannoni, and C. Schmit, Phys. Rev. Lett. 52, 1 (1984)

[6] M. V. Berry and M. Tabor, Proc. R. Soc. London A 356, 375 (1977).

[7] G. E. Mitchell and J. F. Shriner Jr., Phys. Scr. T90, 105 (2001), and references cited therein.

[8] J. B. French, V. K. B. Kota, A. Pandey, and S. Tomsovic, Ann. Phys. (NY) 181, 198 (1988). 
[9] T. Guhr and H. A. Weidenmüller, Ann. Phys. (NY) 199, 412 (1990).

[10] J. F. Shriner Jr., G. E. Mitchell, and T. von Egidy, Z. Phys. A 338, 309 (1991).

[11] J. M. G. Gómez, R. A. Molina, A. Relaño, and J. Retamosa, Phys. Rev. E 66, 036209 (2002).

[12] T. A. Brody, J. Flores, J. B. French, P. A. Mello, A. Pandey, and S. S. M. Wong, Rev. Mod. Phys. 53, 385 (1981).

[13] V. Zelevinsky, B. A. Brown, N. Frazier, and M. Horoi, Phys. Rep. 276, 85 (1996).
[14] R. A. Molina, J. M. G. Gómez, and J. Retamosa, Phys. Rev. C 63, 014311 (2000).

[15] R. A. Molina, Eur. Phys. J. A 28, 125 (2006).

[16] F. J. Dyson and M. L. Mehta, J. Math. Phys. 4, 701 (1963).

[17] J. F. Shriner Jr. and G. E. Mitchell, Z. Phys. A 342, 53 (1992).

[18] D. Mulhall, Z. Huard, and V. Zelevinsky, Phys. Rev. C 76, 064611 (2007).

[19] D. Mulhall, Phys. Rev. C 80, 034612 (2009).

[20] O. Bohigas and M. J. Giannoni, Ann. Phys. (NY) 89, 393 (1975). 